\title{
Constrained Optimization of Linear Antenna Arrays using Novel Social Group Optimization Algorithm
}

\author{
B.Narasimha Rao, S.Saida Rao
}

\begin{abstract}
Antenna array optimization is a major research problem in the field of electromagnetic and antenna engineering. The optimization typically involves in handling several radiation parameters like Sidelobe level $(S L)$ and beamwidth $(B W)$. In this paper, the linear antenna array $(L A A)$ configuration is considered with symmetrical distribution of excitation and special distribution. The objective of the design problem considered involves in generating optimized patterns in terms of SLL and BW and check the robustness of the social group optimization algorithm (SGOA). The analysis of the design problem is carried out in terms of radiation pattern plots. The simulation is carried out in Matlab.
\end{abstract}

Keywords: Antenna array, optimization, SGOA

\section{INTRODUCTION}

The antenna array configuration (AAC) has several advantages over single element antenna. It is possible to obtain high gain and directivity using AAC. It is also possible to direct the beam to any direction of interest using the AAC [1-6]. In addition to beam steering capabilities, it is also possible to shape the radiation pattern in the desired form in order to compete with several applications. Some of the typical applications are the RADAR, cellular and mobile, and several other personal communication systems. All these applications need a variety of radiation patterns which are generally not possible with the single element antenna configuration. Hence, the AAC is preferred to solve such electromagnetic problems.

The AAC can be of several geometrical forms like linear, planar as one dimensional and two dimensional respectively along with three dimensional structures. The synthesis of antenna arrays is an optimization problem as it involves in handling several conflicting parameters of antenna arrays which have to either minimised or maximised [2]. This minimisation or maximization of antenna parameters in the presence of some constraints is always a challenging problem since the inception of the AAC. Several conventional and highly complex numerical methods are suggested to handle the synthesis problem. However, all the conventional problems are typically local search methods. They often have the tendency to give local optimization solution rather than

global competent solution $[2,4,5]$.

Considering the above issues with conventional and

B.Narasimha Rao, Assoc. Professor, Newton's Institute of Science \& Technology, Macherla, Guntur dist. Andhra Pradesh, India.

S.Saida Rao, Assoc. Professor, Newton's Institute of Science \& Technology, Macherla, Guntur dist, Andhra Pradesh, India.

traditional numerical methods, several evolutionary computing algorithms are proposed to antenna array optimization (AAO) problems. Earlier the most famous genetic algorithm, Taguchi method, and particle swarm optimization algorithms are applied successfully to produce optimum sidelobe level (SL) with several constraints. In the recent past, several nature inspired algorithms like flower pollination, firefly, ant colony and bacterial foraging are applied to AAO problems. All the algorithms have produced excellent solutions to the AAO problems. In this paper, a more recent algorithm known as social group optimization algorithm (SGOA) is used for AAO and design. The algorithm is applied to linear array optimization using amplitude-spacing (AmpSp) technique. Further, the paper is written in four Sections. The Section-2 gives a brief introduction to the problem formulation and the description of the algorithm is given in Section-3. The results and discussions are mentioned in Section-4. Overall conclusions of the work are presented in Section 5.

\section{PROBLEM FORMULATION}

The geometrical representation of the structure of the linear array is given in Fig. 1. The presented geometry of the linear array has a symmetrical structure. According to the symmetry the linear array has similar distribution of amplitudes of current excitation and spacing between the elements on either sides of the array.

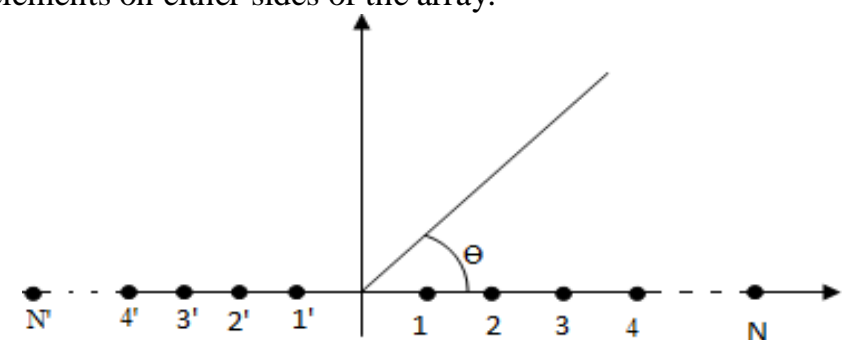

Fig 1: Geometry of the linear array 


\section{CONSTRAINED OPTIMIZATION OF LINEAR ANTENNA ARRAYS USING NOVEL SOCIAL GROUP OPTIMIZATION ALGORITH004D}

The formulation of the array factor is given as [1]

$$
\mathrm{AF}=\sum_{m=0}^{N-1} e^{\mathrm{jm} \psi}
$$

Here

$$
\begin{aligned}
& \psi=\beta d \cos \theta+\alpha \\
& \text { and } \beta=2 \pi / d \\
& \mathrm{~d}=\text { inter-element spacing } \\
& \theta=\text { look angle } \\
& \alpha=\text { phase }
\end{aligned}
$$

The corresponding fitness function is given as

$$
f=\operatorname{Min}\left\{\operatorname{Max}[S L L]_{F N}^{\pi / 2}\right\}
$$

Here, the SL is the sidelobe level in $\mathrm{dB}$ and the first null is represented by FN.

\section{RESULTS \& DISCUSSIONS}

The SGOA is inspired by the social behavior of the human beings belonging to a group or society $[7,8]$. While solving a problem of an individual, the corresponding individual either uses his own personal traits or consults the knowledge of the other members of the society who are called the fellow human beings. In this way the local search and the global search techniques are characterized. However, the potential of the algorithm lies in using the convenient utilization of both the searches in searching for better solution. The two phases of the algorithm are typically known as improvement and acquisition stages. The mathematical modeling of these two stages are given as

Improvement phase:

$$
Y_{a}(i+1)=A^{*} Y_{a}(i)+r *\left[\text { Ybest }_{a}(i)-Y_{a}(i)\right]
$$

Knowledge Acquisition Phase:

$$
\begin{aligned}
& Y_{a(t+1)=} Y_{a(t)}+r_{1}^{*}\left(Y_{a}(i)-Y_{b}(i)\right)+r_{2}\left(\text { Ybest }(i)-Y_{a}(i)\right) \text { if } f\left(Y_{a}(i)\right)<f\left(Y_{b}(i)\right) \\
& Y_{a(t+1)=} Y_{a(t)}+r_{1}^{*}\left(Y_{b}(i)-Y_{a}(i)\right)+r_{2}\left(Y b e s t(i)-Y_{a}(i)\right) \text { if } f\left(Y_{a}(i)\right)>f\left(Y_{b}(i)\right)
\end{aligned}
$$

Here $\mathrm{Y}_{\mathrm{a}}$ is the $\mathrm{a}^{\text {th }}$ individual representing the design variable of the problem. The corresponding iteration is ' $i$ '. The best of the iteration ' $i$ ' is given as $Y_{\text {best }}$. Some uniform distribution random variables are used in the computation which are represented as $\mathrm{A}, \mathrm{r}_{1}$, and $\mathrm{r}_{2}$.

\section{RESULTS:}

The results pertaining to the implementation of the SGOA algorithm for LAA optimization are presented in this Section. The simulation framework is designed to demonstrate the robustness of the algorithm in antenna optimization of different lengths and number of elements. For simulation three different LAA of 12, 20, and 30 elements are considered. However, the corresponding inter-element spacing in all the three cases is considered to be $\lambda / 2$. In all the cases, the non-uniform amplitude distribution of current excitation is determined with respect to the objective of low SLL when compared with those of the uniform distribution. The results are presented in three cases as follows.

\section{Case-1:}

In this case, an LAA of $\mathrm{N}=12$ is considered for synthesis of radiation pattern with suppressed SLL. The uniform LAA has a SLL of $-13.1 \mathrm{~dB}$ which is evident from the radiation pattern plot for the same as shown in Fig. 2 while the corresponding
BW being $19.3^{\circ}$. Now, the SGOA is used to obtain the non-uniform amplitude distribution which produces radiation patterns with SLL suppressed to a level better than those of the uniform distribution. The non-uniform distribution as determined by the SGOA could successfully produce SLL of $-15.9 \mathrm{~dB}$ with the constraint of BW. It means, the SLL suppression is improved by $2.7 \mathrm{~dB}$ while the $\mathrm{BW}$ is unchanged. The radiation pattern plot for this non-uniform distribution is as shown in Fig.3.

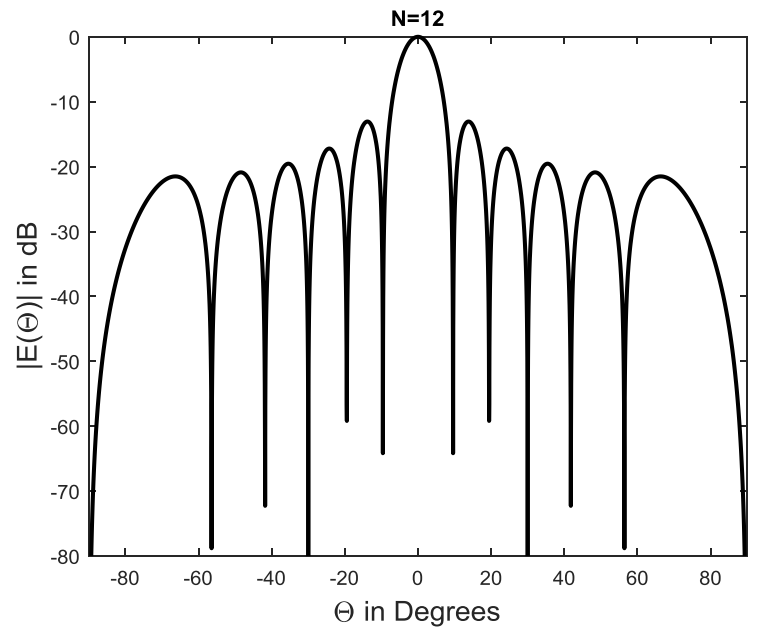

Fig.2: Radiation pattern of LAA with $\mathrm{N}=12$.

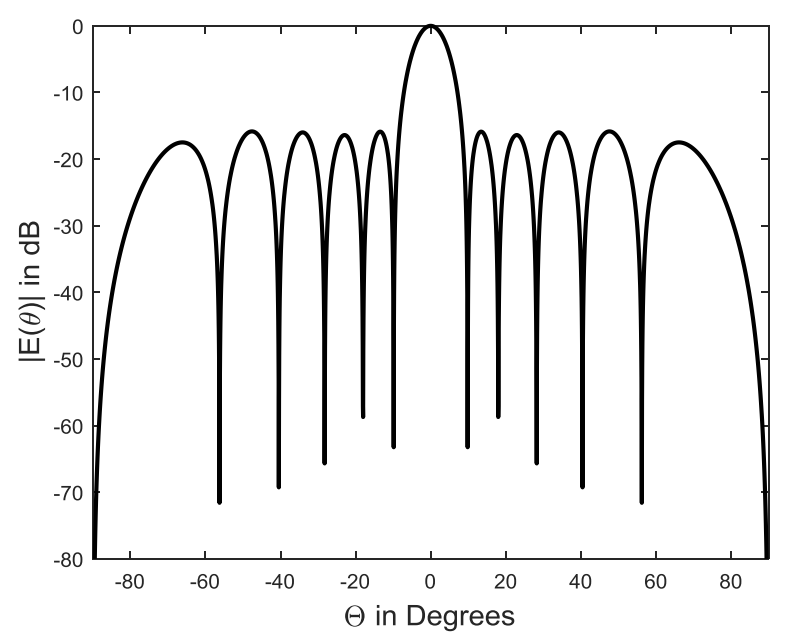

Fig.3: Radiation pattern of non-uniform LAA with $\mathrm{N}=12$ as obtained by SGOA.

\section{Case-2:}

In this case, a LAA of $\mathrm{N}=20$ is considered. The corresponding radiation pattern plot with uniform distribution is as shown in Fig.4. The respective SLL and BW of the uniform distribution of $\mathrm{N}=20 \mathrm{LAA}$ is $-13.18 \mathrm{~dB}$ and $\mathrm{BW}=11.6^{0}$. The radiation patterns pertaining to the non-uniform distribution as determined by the SGOA is also given in Fig.5. The produced radiation patterns have optimized SLL of $-15.9 \mathrm{~dB}$ which is less than that of the uniform distribution. The optimization of the SLL also include the constraint on the corresponding $\mathrm{BW}$ which is 
maintained at $11.6^{\circ}$ as similar as that of the uniform distribution.

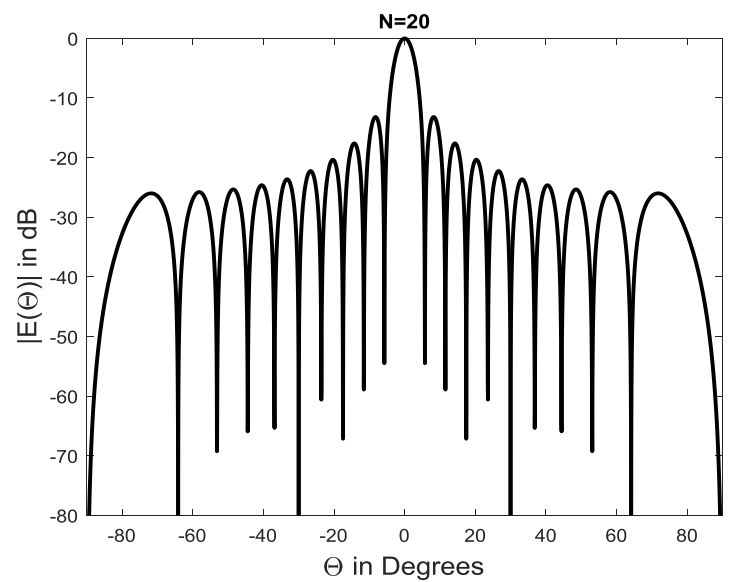

Fig.4: Radiation pattern of LAA of $\mathrm{N}=20$ elements.

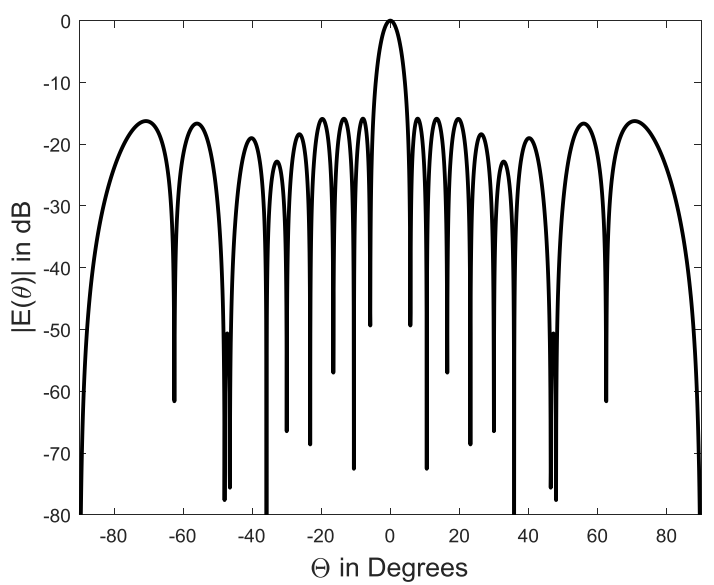

Fig.5: SGOA Optimized LAA of $\mathrm{N}=20$ elements.

\section{Case-3:}

When compared to the previous two cases, the third case has increased the size of LAA to $\mathrm{N}=30$. The corresponding SLL and BW are $-13.2 \mathrm{~dB}$ and $7.7^{\circ}$ respectively as shown in Fig.6. The SGOA is used to determine the non-uniform amplitude distribution for this LAA to optimize the SLL with constraint of BW. The so obtained optimized patterns are as shown in Fig.7.

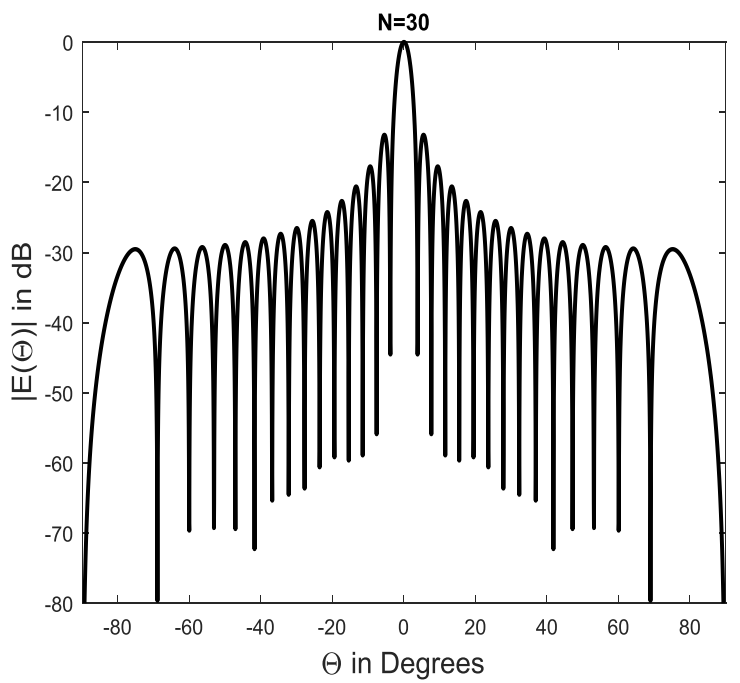

Fig.6: Radiation pattern of uniform LAA of $N=30$ elements.

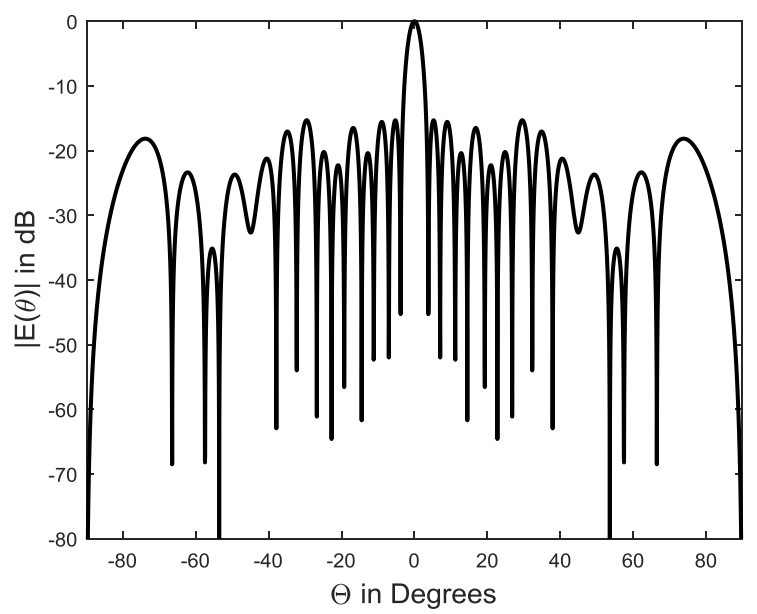

Fig.7: SGOA optimized LAA of $\mathrm{N}=30$ elements

The non-uniform amplitude distribution of the optimized patterns of all the above three cases are listed in Table 1. These amplide coefficients can be used to reproduce the plots of the linear arrays in Fig.3, Fig.5 and Fig.7.

Table.1: SGOA Optimized non-uniform amplitude distribution of LAA

\begin{tabular}{|c|l|c|l|}
\hline $\mathrm{N}$ & \multicolumn{1}{|c|}{ Optimized amplitude distribution (symmetric) } & $\begin{array}{c}\text { SLL (in } \\
\mathrm{dB} \text { ) }\end{array}$ & $\begin{array}{c}\mathrm{BW} \text { (in } \\
\text { degrees) }\end{array}$ \\
\hline 12 & $0.704,0.662,0.656,0.521,0.477,0.779$ & -15.8 & 19.3 \\
\hline 20 & $0.679,0.448,0.708,0.579,0.450,0.553,0.287,0.598,0.469,0.784$ & -15.9 & 11.6 \\
\hline 30 & $\begin{array}{l}0.691,0.832,0.537,0.537,0.552,0.462,0.819,0.538,0.398,0.485, \\
\\
0.819,0.251,0.516,0.734,0.938\end{array}$ & -15.4 & 7.7 \\
\hline
\end{tabular}

\section{CONCLUSION:}

The SGOA algorithm is effectively implemented to optimize the linear array antennas. The objective of constrained optimization of the LAA is achieved with suppressed SLL as low as $-15.9 \mathrm{~dB}$. The consistency of the technique and robustness of the algorithm is evident from the results. Further, the SGOA can be extended to implementation of other antenna optimization techniques involving other design variables in terms of non-uniform special distribution of the elements of the array in the geometry.

\section{REFERENCES:}

1. Balanis, C. A., Antenna Theory: Analysis and Design, John Wileyand Sons, 1982

2. Cheng, K. D: Optimization techniques for antenna arrays. In: Proceedings of the IEEE, 59(12) 1664-1674 (1971).

3. On the Linear Antenna Array Synthesis Techniques for Sum and Difference Patterns Using Flower Pollination Algorithm V. V. S. S. S. Chakravarthy - P. S. R. Chowdary · Ganapati Panda ·Jaume Anguera · Aurora Andújar - Babita Majhi Proceedings of Arabian Journal for Science and Engineering - Springer Nature Hub.

4. Ram, G.; Mandal, D.; Ghoshal, S.P.; Kar, R.: Nature-inspired algorithm- based optimization for beamforming of linear antenna array system. In: Patnaik, 
S. et al. (eds.) Nature-Inspired Computing and Optimization 2017, pp. 185-215. Springer, Berlin. doi:10.1007/978-3-319-50920-4_8

5. Performance of Beamwidth Constrained Linear Array Synthesis Techniques Using Novel Evolutionary Computing Tools CSR Paladuga, CV Vedula, J Anguera, RK Mishra, AAndújar, applied computational electromagnetics society journal 33 (3),273-278

6. Saxena, P.; Kothari, A.: Linear Antenna Array Optimization Using Flower Pollination Algorithm. Springer, Berlin(2016).

7. Suresh Satapathy and Anima Naik.:Social group optimization (SGO): a new population evolutionary optimization technique. In: Complex Intell. Syst., (2) 173-203 (2016).

8. Antenna Array Synthesis Using Social Group Optimization VS Chakravarthy, PSR Chowdary, SC Satpathy, SK Terlapu, J Anguera Microelectronics, Electromagnetics and Telecommunications,895-905. 